\title{
OPERATOR INEQUALITIES AND GYROLINES OF THE WEIGHTED GEOMETRIC MEANS
}

\begin{abstract}
SEJONG KIM
Abstract. We consider in this paper two different types of the weighted geometric means of positive definite operators. We show the component-wise bijection of these geometric means and give a geometric property of the spectral geometric mean as a metric midpoint. Moreover, several interesting inequalities related with the geometric means of positive definite operators will be shown. We also see the meaning of weighted geometric means in the gyrogroup structure with finite dimension and find the formulas of weighted geometric means of 2-by-2 positive definite matrices and density matrices.
\end{abstract}

Mathematics subject classification (2010): 47B65, 15B48.

Keywords and phrases: Positive definite operator, Loewner order, geometric mean, spectral geometric mean, gyrogroup, gyroline.

\section{REFERENCES}

[1] E. Ahn, S. Kim, AND Y. Lim, An extended Lie-Trotter formula and its applications, Linear Algebra Appl. 427 (2007), 190-196.

[2] T. ANDO, Geometric mean and norm Schwarz inequality, Ann. Func. Anal. 7 (2016), no. 1, 1-8.

[3] T. ANDo AND F. HiAI, Log majorization and complementary Golden-Thompson type inequalities, Linear Algebra Appl. 197 (1994), 198: 113-131.

[4] R. Bhatia, Matrix Analysis, Springer, 1997.

[5] R. Bhatia, Positive Definite Matrices, Princeton Series in Applied Mathematics, Princeton University Press, 2007.

[6] R. Bhatia AND J. HolBRook, Riemannian geometry and matrix geometric means, Linear Algebra Appl. 413 (2006), 594-618.

[7] G. Corach, H. Porta, And L. Recht, Convexity of the geodesic distance on spaces of positive operators, Illinois J. Math. 38 (1994), 87-94.

[8] M. FIEDlER AND V. PTÁK, A new positive definite geometric mean of two positive definite matrices, Linear Algebra Appl. 251 (1997), 1-20.

[9] Y. Friedman And T. SCARR, Physical Applications of Homogeneous Balls, Birkhäuser, Basel, 2005.

[10] R. A. Horn and C. R. Johnson, Matrix Analysis, 2nd edition, Cambridge University Press, 2013.

[11] H. KARCHER, Riemannian center of mass and mollifier smoothing, Comm. Pure Appl. Math. 30 (1977), no. 5, 509-541.

[12] S. KIM, Distances of qubit density matrices on Bloch sphere, J. Math. Phys. 52 (2011), 102303.

[13] S. KIM, Distributivity on the gyrovector spaces, Kyungpook Math. J. 55 (2015), 13-20.

[14] S. KIM, Gyrovector spaces on the open convex cone of positive definite matrices, Mathematics Interdisciplinary Research 1 (2016), 173-185.

[15] S. Kim, S. Kim, AND H. LeE, Factorizations of invertible density matrices, Linear Algebra Appl. 463 (2014), 190-204.

[16] S. Kim AND H. LEE, Relative operator entropy related with the spectral geometric mean, Anal. Math. Phys. 5, Vol. 3 (2015), 233-240.

[17] F. Kubo And T. Ando, Means of positive linear operators, Math. Ann. 246 (1979/80), no. 3, 205224. 
[18] J. Lawson And Y. Lim, The geometric mean, matrices, metrics, and more, Amer. Math. Monthly 108 (2001), 797-812.

[19] J. Lawson AND Y. Lim, Metric convexity of symmetric cones, Osaka J. Math. 44 (2007), 795-816.

[20] J. LAWSON AND Y. LIM, Karcher means and Karcher equations of positive definite operators, Trans. Amer. Math. Soc. Series B, Vol. 1 (2014), 1-22.

[21] H. LEE AND Y. Lim, Metric and spectral geometric means on symmetric cones, Kyungpook Math. J. 47 (2007), 133-150.

[22] Y. LiM, Geometry of midpoint sets for Thompson's metric, Linear Algebra Appl. 439 (2013), 211-227.

[23] J. S. MATharu AND J. S. Aujla, Some inequalities for unitarily invariant norms, Linear Algebra Appl. 436 (2012), 1623-1631.

[24] M. MOAKHER, A differential geometric approach to the geometric mean of symmetric positive-definite matrices, SIAM J. Matrix Anal. Appl. 26 (2005), no. 3, 735-747.

[25] R. D. Nussbaum, Finsler structures for the part metric and Hilbert's projective metric and applications to ordinary differential equations, Differential and Integral Equations 7 (1994), 1649-1707.

[26] W. Pusz, AND S. L. Woronowicz, Functional calculus for sesquilinear forms and the purification map, Rep. Math. Phys. 8 (1975), 159-170.

[27] A. C. THOMPSON, On certain contraction mappings in a partially ordered vector space, Proc. Amer. Math. Soc. 14 (1963), 438-443.

[28] M. UchiYama, Criteria for monotonicity of operator means, J. Math. Soc. Japan, 53 (2003), 197207.

[29] A. A. UngaR, Analytic hyperbolic geometry and Albert Einstein's special theory of relativity, World Scientific Press, 2008.

[30] T. YAMAZAKI, The Riemannian mean and matrix inequalities related to the Ando-Hiai inequality and chaotic order, Oper. Matrices 6 (2012), 577-588.

[31] F. Zhang, Matrix Theory: Basic Results and Techniques, 2nd edition, Springer, 2011.

[32] K. ZHU, Spaces of Holomorphic Functions in the Unit Ball, Graduate Texts in Mathematics, Springer, New York, 2005. 